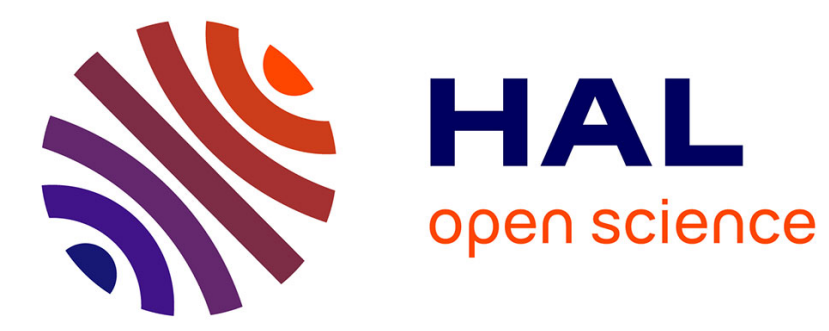

\title{
Investigation of gelation phenomena of some polysaccharides by ultrasonic spectroscopy
}

Michel Audebrand, Jean-Louis Doublier, D. Durand, Jacques R. Emery

\section{To cite this version:}

Michel Audebrand, Jean-Louis Doublier, D. Durand, Jacques R. Emery. Investigation of gelation phenomena of some polysaccharides by ultrasonic spectroscopy. Food Hydrocolloids, 1995, 9 (3), pp.195-203. 10.1016/S0268-005X(09)80216-6 . hal-02706398

\section{HAL Id: hal-02706398 \\ https://hal.inrae.fr/hal-02706398}

Submitted on 25 Aug 2021

HAL is a multi-disciplinary open access archive for the deposit and dissemination of scientific research documents, whether they are published or not. The documents may come from teaching and research institutions in France or abroad, or from public or private research centers.
L'archive ouverte pluridisciplinaire HAL, est destinée au dépôt et à la diffusion de documents scientifiques de niveau recherche, publiés ou non, émanant des établissements d'enseignement et de recherche français ou étrangers, des laboratoires publics ou privés.

\section{(c)(1)}

Distributed under a Creative Commons Attribution| 4.0 International License 


\title{
Investigation of gelation phenomena of some polysaccharides by ultrasonic spectroscopy
}

\author{
M.Audebrand, J.L.Doublier, D.Durand ${ }^{1}$ and J.R.Emery' \\ I.N.R.A., rue de la Géraudière, 44026 Nantes and ${ }^{1}$ URA CNRS 509 and 807 Université du Maine, route de Laval, \\ 72017 Le Mans, France
}

\begin{abstract}
The gelation process of two polysaccharides, amylose and alginate, has been investigated by means of ultrasonic spectroscopy. This method allows an immediate analysis of the acoustic wave propagating through the medium by means of Fast Fourier Transform of the signals. Measurements were performed within the frequency range 15-100 $\mathrm{MHz}$. The absorption and the velocity of the ultrasonic wave can therefore be obtained as a function of time over a given frequency range. The results were compared to classical rheological measurements in oscillatory shear at a low strain amplitude and, in case of amylose, to turbidity variations. In the course of amylose gelation, variations in the absorption of the ultrasonic signal paralleled those of turbidity in the medium and took place at a much earlier stage than the one at which the increase in $\mathrm{G}^{\prime}$ and $\mathrm{G}^{\prime \prime}$ can be detected. In the case of alginate, absorption variations paralleled more closely those of the viscoelastic parameters $\mathrm{G}^{\prime}$ and $\mathrm{G}^{\prime \prime}$. The differences can be interpreted in relation to the mechanisms of gelation. Amylose gelation takes place through a phase separation. Ultrasound appears extremely sensitive to such changes in the material. The method shows the first steps in the phase separation phenomenon. This suggests that variations in the ultrasonic absorption can be taken as a means to detect the earlier steps of the gelation process of starchy materials. Gelation of alginate is a calcium-mediated process, in which case, ultrasounds provide similar information as rheological measurements.
\end{abstract}

\section{Introduction}

The physical characterization of food gelling systems often implies rheological measurements. Even if they are carried out at very low stress and strain amplitude, the disruption in the structure of the material being investigated may be significant. For this reason, ultrasound has been proposed as an alternative to rheological measurements. The propagation of ultrasonic waves through a material creates mechanical vibration inside it, and therefore, stress and strain. It is well known that high power ultrasound can yield breakdown at a molecular level, being therefore unsuitable. However, at a low ultrasonic power level, the medium is not broken down or disturbed, the amplitude of the strain being close to molecular scale. Ultrasound has been employed in several fields of food science. A former comprehensive literature survey of the applications of ultrasound in food systems has been published (1). The author reviewed ultrasonic techniques which had been applied to food systems and discussed the problems encountered. On another hand, Povey and McClements (2) reviewed the various experimental methods available for the use of ultrasound in food engineering. More recently, this group $(3,4)$ measured ultrasonic velocity and attenuation on emulsions. For example, they determined the melting and crystallization temperatures of these food systems. The proportion of droplets which had crystallized and the relaxation mechanisms associated with the phase transitions could be evaluated. Also, an ultrasonic method has been developed to monitor gravitational sedimentation of particles in a dispersion (5). Particle size distribution could be obtained from the concentration profile. Recently, it has been shown that rheological properties of cheese and dough can be evaluated using shear ultrasonic waves (6) and the authors found a good correlation with data obtained from a traditional rheometer. All these different experimental methods can be applied to on-line measurements. Ultrasonic methods were also used to investigate the 
dynamics of molecular processes. For example, some results were reported concerning ultrasonic relaxation of polysaccharides in the sol and gel states (7-11). A slight excess ultrasonic attenuation was observed for carrageenan sol ( $\mathrm{Na}, \mathrm{K}, \mathrm{Ca}$, tetramethylammonium) salts $<10 \mathrm{MHz}$ and was ascribed to a counterion binding relaxation mechanism (9). On another hand, no relaxation mechanism between 1 and $100 \mathrm{MHz}$ was reported for dextran solutions (11). In contrast, from the ultrasonic relaxation spectra of agarose and carrageenan gels, a strong relaxation process at low frequencies $(<10 \mathrm{MHz})$ was observed arising from the motion of the solvent in the molecular network $(8,10)$.

The object of this paper was to demonstrate the feasibility of ultrasonic techniques in characterizing the solgel transition in polysaccharide systems. Information related to the polymer-solvent or polymer-polymer interactions at each time point during the gelation process was sought which would lead to a better understanding of the gelling process. Two types of polysaccharide networks known to gel through different processes were investigated: amylose gels and alginate gels. Amylose gelation takes place through a phase separation process between polymerrich and polymer-deficient phases $(12,13)$. A crystallization of amylose chains within the polymer rich-phase then occurs, which has been shown as a secondary process. In contrast, the gelation process of alginate gels occurs through calcium chelation between two polyguluronic chains displaying a regular array of electronegative cavities. This mechanism has been described by the well known 'eggbox' model $(14,15)$. Aggregation of dimers is then possible yielding the gel network. In order to observe slow gelation, low concentration solutions (1-1.5\% for amylose, $0.5 \%$ for alginate) were studied. An ultrasonic spectroscopic method was used to describe the frequency-dependent properties of the material. This technique has already been employed to monitor the gelation of synthetic polymers such as epoxy resins (16). A similar method which has been called 'frequency scanning ultrasonic pulse echo reflectometer' (FSUPER) has been used to characterize oil-in-water emulsions and to examine the effect of $\mathrm{pH}$ and ionic strength on globular proteins (17). A fast Fourier transform analysis of a pulse transmitted through the medium allowed us to describe the evolution of the system in real time over a range of frequency. In parallel, rheological and turbidity measurements were performed.

\section{Materials and methods}

\section{Amylose}

The amylose, purchased from Avebe (The Netherlands), was from potato starch. Its average molecular weight $\left(\mathbf{M}_{\mathbf{w}}\right)$ as measured from light scattering in $0.1 \mathrm{~mol} / \mathrm{dm}^{3} \mathrm{KCl}$ was of the order of 200000 . The amylose sample was purified by dispersion in DMSO $\left(70^{\circ} \mathrm{C}\right.$ for $\left.30 \mathrm{~min}\right)$, followed by precipitation by ethanol addition. After filtration the resulting powder was dried and stored.

\section{Alginate}

The alginate sample of sodium (SG150) was supplied by Sanofi Bio Industrie (Carentan, France). It had been extracted from Laminaria Hyperborea. Its guluronic content was quite high (mannuronic/guluronic $\sim 0.6$ ).

\section{Preparation of gels}

\section{Amylose}

The amylose powder was dispersed in $1 \mathrm{~mol} / \mathrm{dm}^{3} \mathrm{KOH}$ to obtain an $\sim 9 \%(w / w)$ solution. This stock solution was stored at $4^{\circ} \mathrm{C}$. Gels were prepared under isothermal conditions by adding 1 or $0.3 \mathrm{~mol} / \mathrm{dm}^{3} \mathrm{HCl}$ dropwise to the solution of amylose as described in (18). The final concentration (1.03-1.45\%) and ionic strength $\left(0.3 \mathrm{~mol} / \mathrm{dm}^{3} \mathrm{KCl}\right)$ were calculated from the quantity of components which had been added to obtain a $\mathrm{pH}$ of 7 .

\section{Alginate}

Alginate can gel through a progressive release of calcium ions initially complexed with EDTA as EDTA-Na $-\mathrm{Ca}$. Addition of glucono- $\delta$-lactone (GDL) induces a slow decrease of the $\mathrm{pH}$ therefore resulting in the freeing of calcium ions. Stock solutions of alginate and EDTA- $\mathrm{Na}_{2}-$ Ca were prepared. A $8 \%$ solution of GDL was added in appropriate amounts to mixture of these two solutions in order to yield the following composition: $0.5 \%$ alginate, $0.5 \%$ EDTA- $\mathrm{Na}_{2}-\mathrm{Ca}$, GDL ranging from 0.35 to $0.5 \%$. The origin of the time was taken as the time when GDL was added. In order to avoid sound scattering, in the case of amylose and alginate gelling, the presence of air bubbles was reduced by slow mixing of the solutions.

\section{Rheology}

Small-amplitude oscillatory-shear experiments were performed at $25^{\circ} \mathrm{C}$ using a Rheometrics Fluid Spectrometer (RFSII) with a cone-plate geometry $\left(5 \mathrm{~cm}\right.$ diameter; $1^{\circ}$ cone angle). Measurements were carried out at $1 \mathrm{rad} / \mathrm{s}$ and at a strain amplitude of $5 \%$.

\section{Turbidity}

Turbidity was monitored by measuring absorbance variations as a function of time at $600 \mathrm{~nm}$ and $25^{\circ} \mathrm{C}$ using a u.v.l visible spectrophotometer.

\section{Ultrasonic spectroscopy}

\section{General}

The velocity and attenuation of an acoustic wave travelling through a medium depends on the mechanical properties of the medium which can be described by different moduli according to the type of deformation. If pure shear modes are stimulated, one can measure the real and imaginary part of the complex shear modulus $G^{*}$ which can be directly compared to rheological measurements. Unfortunately, shear waves in a viscoelastic liquid are strongly attenuated, so that they propagate only a few microns from their source 
of excitation. This is not the case for longitudinal waves. If longitudinal modes travel through a viscoelastic medium, both shear and compressional effects appear simultaneously and can be expressed as a sum of two moduli:

$$
M^{*}=K^{*}+\frac{4}{3} G^{*}=M^{\prime}+i M^{\prime \prime}
$$

where $M^{*}$ is the complex longitudinal modulus and $K^{*}$ is the complex bulk modulus.

The real part $M^{\prime}$ and imaginary part $M^{\prime \prime}$ of the longitudinal modulus $M^{*}$ can be determined by measuring the longitudinal absorption $\alpha$ and the velocity $V_{l}$ such as:

$$
M^{\prime}=K^{\prime}+\frac{4}{3} G^{\prime}=\rho V_{l}^{2} \quad M^{\prime \prime}=K^{\prime \prime}+\frac{4}{3} G^{\prime \prime}=2 \rho V_{l}^{3} \frac{\alpha}{\omega}
$$

Here $\rho$ is the density and $\omega=2 \pi f$ the angular frequency. The velocity dispersion and a certain part of the attenuation in a viscoelastic liquid depend on molecular relaxation mechanisms. It is to be noticed that the shear moduli, $G^{\prime}$ and $G^{\prime \prime}$, can easily be obtained from rheological measurements but at relatively low frequency. It is obvious that shear moduli obtained within this frequency range $\left(10^{-2}-\right.$ $10 \mathrm{~Hz}$ ) cannot be compared to those at ultrasonic frequencies $(>1 \mathrm{MHz})$, due to likely relaxation processes in between these two frequency windows. Therefore, a direct comparison of $M^{\prime}$ and $M^{\prime \prime}$, from ultrasonic measurements, and of $G^{\prime}$ and $G^{\prime \prime}$, from rheological measurements, is not possible. For longitudinal waves, two kinds of relaxation processes can exist: a bulk relaxation for $K$ with a relaxation time $\tau_{v}$ and a shear relaxation for $G$ with a relaxation time $\tau_{\mathrm{s}}$. In most cases relaxation effects which involve structural processes have a time distribution. Therefore, the frequency dependence of $M$ is governed by all these processes and their eventual distribution of relaxation times. As for shear waves, if the distribution of relaxation times is broad and continuous, one can expect that the power-law $G^{\prime} \sim G^{\prime \prime} \sim \omega^{\mathrm{n}}$ at the onset of gelation can be applied to the longitudinal modulus $M$. Longitudinal waves are easy to use but without further information, one cannot separate the contribution of shear or bulk. This double contribution increases the difficulty of determining the physical nature of the observed relaxation.

\section{Experimental}

The main difficulty is measuring the frequency dependence of the velocity and attenuation of ultrasound of a dynamic system in real time. To overcome this problem, an ultrasonic signal processing device allowing a frequency analysis of velocity and attenuation has been developed $(19,20)$.

A Biosonic ER100 pulser/receiver was used for excitation and reception of wideband ultrasonic signals. An ultrasonic transducer emits a wideband ultrasonic longitudinal pulse which is sent through the sample under study. The transmitted signal is received by the receiving transducer passed through an amplifier (receiver) and is digitized by a Lecroy 9400A digital oscilloscope providing $175 \mathrm{MHz}$ bandwith with $5 \mathrm{Ghz}$ sample rate. The digitized signal is immediately transferred and processed on a personal computer using ASYST 2.0. In addition to analysis, the experiment was computer-controlled. A thermostated cylindrical support of internal diameter of $1.6 \mathrm{~cm}$ was built to accommodate the ultrasonic transducers. These transducers could be moved axially inside the support to produce a known gap between them. The width of this gap was taken as $1.3 \mathrm{~cm}$ defining the experimental cell which was filled by the sample $(2.6 \mathrm{ml})$.

A Fast Fourier Transform (F.F.T.) of the pulse response was computed to obtain the transfer function of the material. This function describes the mechanical properties of the material in the frequency domain limited by the bandwidth of the transducers. By taking as reference the time $t=0$, the comparison of the amplitude spectrum of the F.F.T. at each time, during the gelling process allowed us to determine the frequency dependence of the relative absorption $\Delta \alpha(\omega)$. In the same way, from the comparison of the phase spectrum the frequency dependence of the relative velocity $\Delta V(\omega)$ was obtained.

$$
\Delta \alpha(\omega)=\frac{1}{l} \ln \frac{U(\omega, 0)}{\mathrm{U}(\omega, t)} \quad \Delta \mathrm{V}(\omega)=\frac{\omega l}{\Phi(\omega, t)-\Phi(\omega, 0)}
$$

Here $U(\omega, 0)$ and $U(\omega, t)$ are, respectively, the magnitudes of the amplitude spectrum at times $t=0$ and $t$ for the frequency $f . l$ is the distance between the transducers. $\Phi(\omega, 0)$ and $\Phi(\omega, t)$ are, respectively, the phases at times $t=$ 0 and $t$ for the frequency $f$. The value $\Phi(\omega, t)-\Phi(\omega, 0)$ modulo $2 \pi$ is obtained from the comparison of the phase spectrum. Since longitudinal moduli, $M^{\prime}$ and $M^{\prime \prime}$, are proportional to $\alpha$ and $v$, respectively, we considered the evolution of these two last parameters directly rather than computing $M^{\prime}$ and $M^{\prime \prime}$. We did not perform any frequency domain correction that may be required as reported by Holmes et al. (21). This was justified by the fact that the instrumental system had been tested previously by comparing the measured values of some test liquids (water, 1-1-2 thrichloroethane, disulfur carbone) to standard values of the literature with an excellent concordance $(<1 \%$ for $\alpha$ and $10^{-4}$ for $v$ in relative values) (20).

Experimental data were acquired at three minute intervals. The time of measurements was the time of acquisition which varied from one millisecond to a few seconds depending on the frequency and on the averaging of the signal. The time of processing was about $10 \mathrm{~s}$. Measurements were carried out with two pairs of transducers with nominal frequency of $\sim 50$ and $120 \mathrm{MHz}$ respectively, giving longitudinal waves and a total frequency bandwidth in the material ranging from 20 to 100 $\mathrm{MHz}$. No dispersion of velocity was observed so that only evolution of attenuation was considered. 


\section{Results and discussion}

\section{Amylose gelation}

Figure 1 shows a typical example of the evolution of the ultrasonic absorption $(\Delta \alpha)$ during the gelation process of a $1.03 \%(\mathrm{w} / \mathrm{w})$ amylose solution in $0.3 \mathrm{~mol} / \mathrm{dm}^{3} \mathrm{KCl}$ at $25^{\circ} \mathrm{C}$. For the three frequencies of this example, namely 50, 80 and $100 \mathrm{MHz}$, two separate experiments had to be carried out. The absorption at 50 and $80 \mathrm{MHz}$ was obtained with the transducers having the $120 \mathrm{MHz}$ nominal frequency. The curve at $25 \mathrm{MHz}$ was obtained with the $50 \mathrm{MHz}$ transducers. It is clearly illustrated that the absorption depends upon frequency, the higher the frequency, the

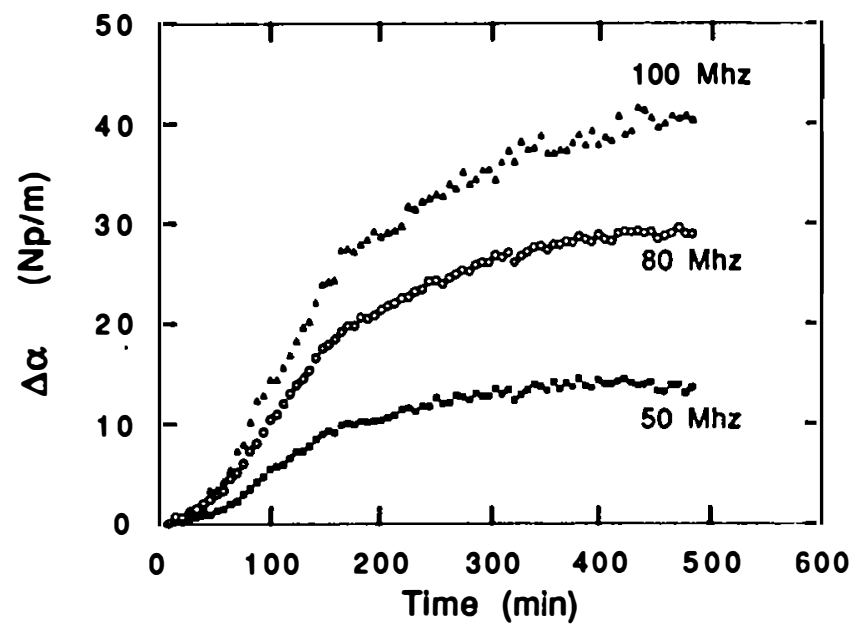

Figure 1 Variations of the absorption, $\Delta \alpha$, as a function of time for a $1.03 \%$ amylose concentration at three different frequencies: 50,80 and $100 \mathrm{MHz}$ (in $0.3 \mathrm{~mol} / \mathrm{dm}^{3} \mathrm{KCl}$; temperature: $25^{\circ} \mathrm{C}$ ).

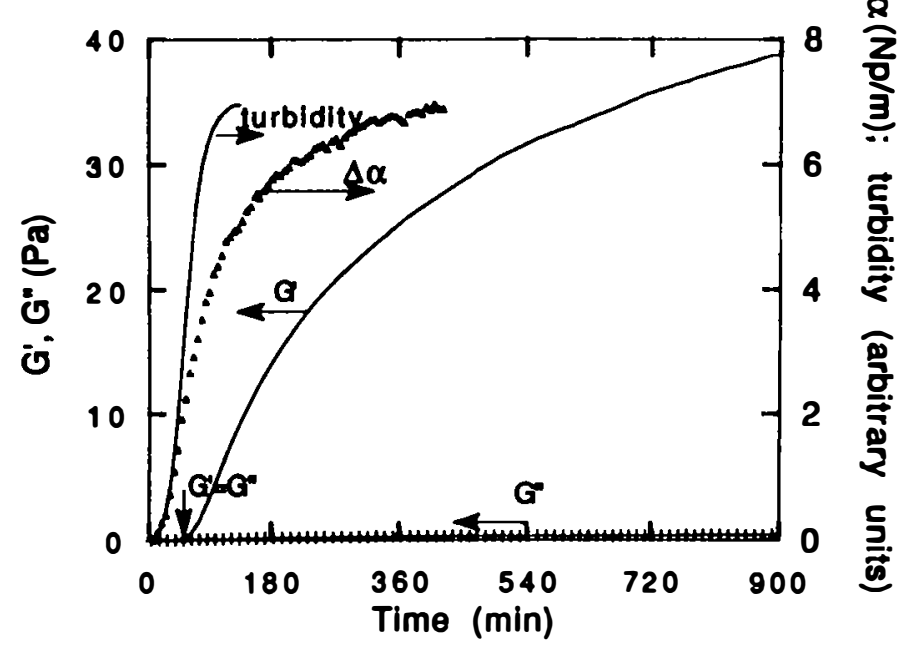

Figure 2 Comparison of $\Delta \alpha$ variations, measured at $25 \mathrm{MHz}$, with the turbidity and the $G^{\prime}$ and $G^{\prime \prime}$ variations $(1.24 \%$ amylose concentration; $0.3 \mathrm{~mol} / \mathrm{dm}^{3} \mathrm{KCl}$ ). Symbols: $\Delta, \Delta \alpha$; $+G^{\prime \prime}$. Continuous lines: $G^{\prime}$ and turbidity. The arrow shows the gel point as defined by $G^{\prime}=G^{\prime \prime}$. stronger the absorption. However, the three curves showed a similar shape. During the first $50 \mathrm{~min}$, the absorption increased slowly; then, a sharp increase took place up to $\sim 150-200 \mathrm{~min}$. Afterwards, the absorption increased steadily without reaching a steady state value even after $10 \mathrm{~h}$.

Meanwhile, the evolution of the shear moduli, $G^{\prime}$ and $G^{\prime \prime}$, and of the turbidity as a function of time were recorded. This is illustrated in Figure 2 together with the absorption and the turbidity variations for a $1.24 \%(\mathrm{w} / \mathrm{w})$ amylose solution. $\Delta \alpha$, measured at $25 \mathrm{MHz}$, increased from 0 to 7 Neper per meter after $450 \mathrm{~min}$. $G^{\prime}$ varied from 0.1 to 39 Pascals after 900 min whereas $G^{\prime \prime}$ remained below $0.4 \mathrm{~Pa}$. For convenience, these values were plotted in normalized coordinates, i.e. each value is divided by its ultimate one (Figure 3). Overall, the four curves exhibited a similar shape characterized by the three main zones as described above. However, it is clear that the $\Delta \alpha$ variations were closer to the turbidity ones than the $G^{\prime}$ and $G^{\prime \prime}$ curves.

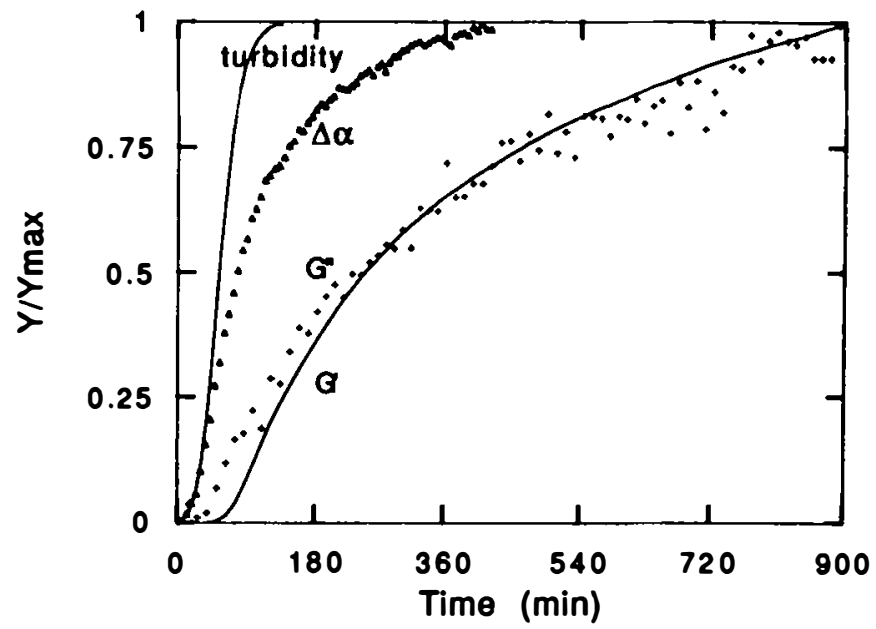

Figure 3 Same results as in Figure 2 plotted in normalized ordinates. Symbols: $\Delta, \Delta \alpha$;,$+ G^{\prime \prime}$. Continuous lines: $G^{\prime}$ and turbidity.

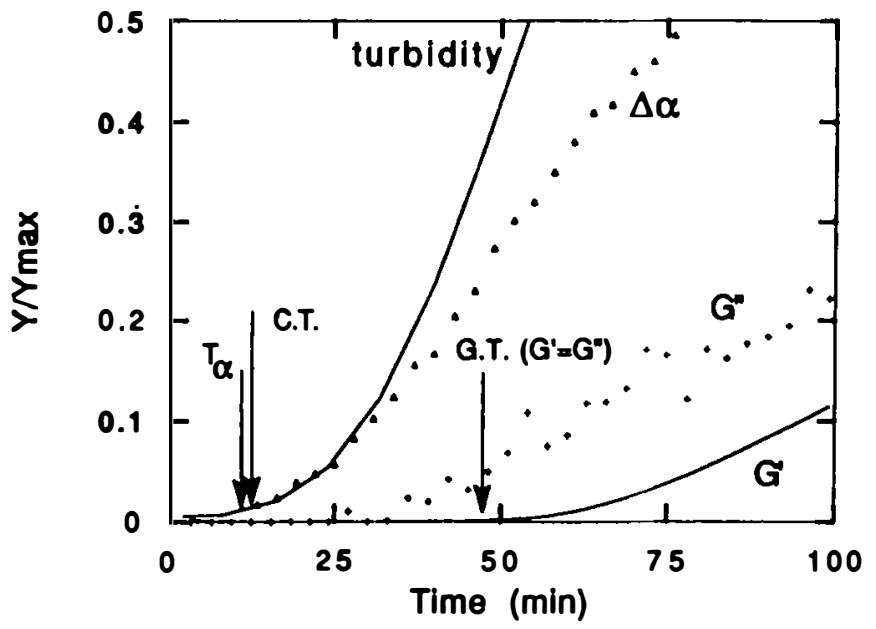

Figure 4 As in Figure 3 for the first $100 \mathrm{~min}$. Arrows show gel time $\left(G^{\prime}=G^{\prime \prime}\right)$, cloud time (C.T.) and $T_{\alpha}$. 
The small values of $G^{\prime \prime}$ plotted in normalized coordinates introduced some noise. The first $100 \mathrm{~min}$ of the process are plotted in an expanded form in Figure 4. This illustrates clearly that for each method a lag time is observed. In rheology, the lag time was defined as the gel time (G.T.) and was taken as the cross-point of the $G^{\prime}$ and $G^{\prime \prime}$ traces. In case of turbidity measurements, this time corresponded to the appearance of cloudiness. It has been defined as the Cloud Time (C.T.) and taken quite arbitrarily as the point of departure from the baseline of the turbidity curve (18). From these two methods, we estimated G.T. at $\sim 48 \mathrm{~min}$ and C.T. at $\sim 15.5 \mathrm{~min}$. Similarly, we defined a lag time from ultrasonic measurements, $T_{\alpha}$, as the point at which $\Delta \alpha$ was $0.025 \mathrm{Neper} / \mathrm{m}$ greater than the first measurement. In the present example, $T_{\alpha}$ was equal to $15 \mathrm{~min}$ and of the order of the C.T.

The gelation process of amylose in low concentration conditions has been shown to take place in three steps $(18,22,23)$. The first step is an induction period when a phase separation takes place, giving rise to polymer-rich and polymer-poor phases. The C.T. has been taken as an estimate of the length of this phase, the higher the concentration in amylose, the lower C.T. The second step, beyond C.T., is characterized by a further aggregation which is probably combined to a crystallization of amylose chains. The third phase is governed by a further crystallization of the amylose chains. In this last phase, diffusion of macromolecules is slowed down due to the high viscosity of the medium. As well as for rheology or turbidity measurements, ultrasonic absorption data monitor these three periods. The formation of aggregates yields a small change in the viscosity of the medium which is probably responsible for the slight increase of $\Delta \alpha$ in the first step. The threedimensional network which then grows in the second step with high change of the viscosity results in the sharp increase of $\Delta \alpha$. In the third phase, the $\Delta \alpha$ increase is slowed down as well as the $G^{\prime}$ increase.

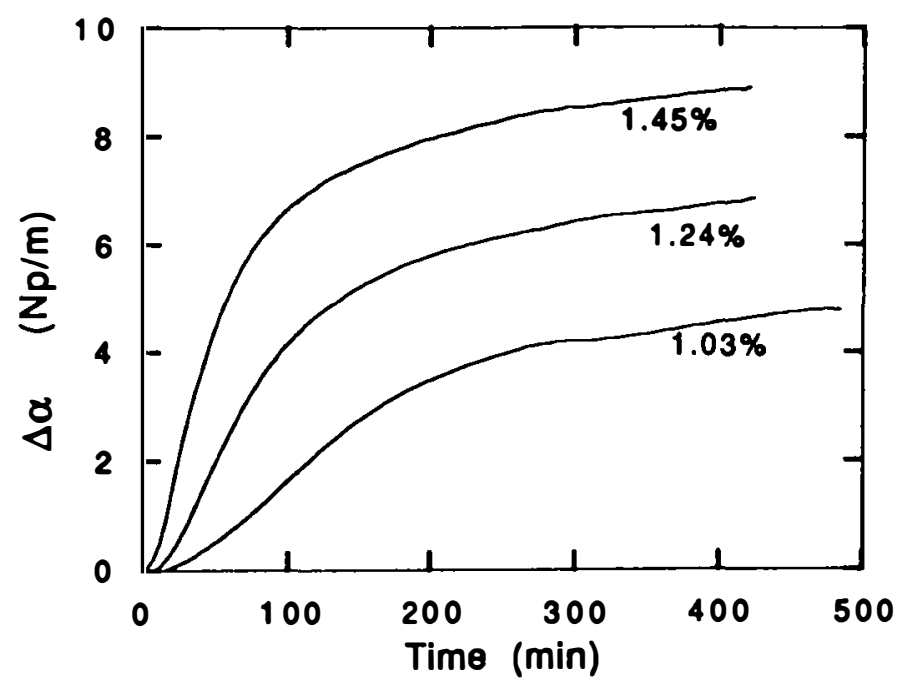

Figure 5 Variations of the absorption $(\Delta \alpha)$ as a function of time for three amylose concentrations: $1.03,1.24$ and $1.45 \%$.
The effect of amylose concentration on ultrasonic absorption is illustrated in Figure 5. When the concentration increased from 1.03 to $1.45 \%(w / w)$, the absorption measured at $25 \mathrm{MHz}$ at the end of the ageing period increased from 4 to $9 \mathrm{Neper} / \mathrm{m}$ while $G^{\prime}$ varied from 1.35 to $85 \mathrm{~Pa}$ and $G^{\prime \prime}$ from 0.02 to $0.65 \mathrm{~Pa}$. It is clear that $\Delta \alpha$ variations were much smaller than those of $G^{\prime}$. On the other hand, $T_{\alpha}$ increased as the concentration decreased. Similar variations were found for the lag times (G.T. and C.T.) determined from rheology and turbidity measurements. This is illustrated in Figure 6 in logarithmic scales. Linear dependencies were found with slope of $\sim-3.4$ for $T_{\alpha}$ and C.T. A line almost parallel (slope: $\sim 4.2$ ) but shifted to higher values could be drawn for the G.T. Therefore, whatever the concentration, there was a ratio almost constant between G.T. and $T_{\alpha}$ (or C.T.), of the order of 3. This correspondence shows that $T_{\alpha}$ is also a characteristic of the gelation process. It is worthy of note that a previous study on another amylose sample (18) has shown a similar dependency of C.T. upon concentration (slope: -4.1). However, a closer correspondence between C.T. and G.T. with C.T./G.T. $\simeq 1.2$ had been reported. The molecular weight of amylose sample in the previous work was twice as high as that studied in the present work, and this may explain the apparent discrepancy. However, the dependence of C.T. (or G.T. or $T_{\alpha}$ ) upon concentration has shown the same correlation, and this confirms that the induction period is strongly concentration-dependent. Actually, the ultrasonic absorption increased slightly from the very beginning of the process. This is consistent with the fact that aggregation of amylose molecules proceeds as soon as neutral $\mathrm{pH}$ is reached. The fact that turbidity only evolved from C.T. is primarily related to the size of aggregates which are too small to be detected by the spectrophotometer below this critical time (18). Similarly, the rheological measurements are only able to detect an evolution beyond given values of $G^{\prime}$ and $G^{\prime \prime}$ due to the

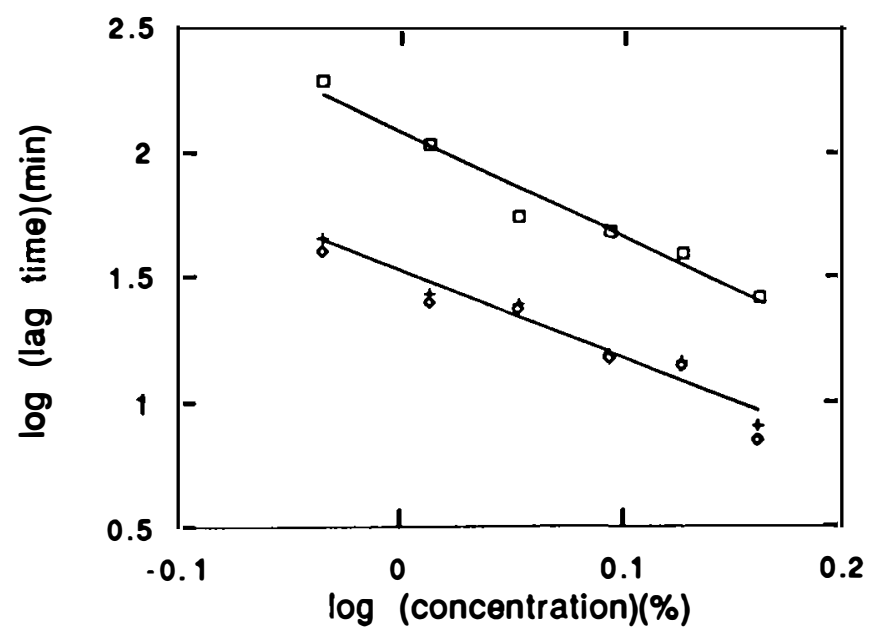

Figure 6 G.T., cloud time and $T_{\alpha}$ variations as a function of concentration: $\square$, G.T.;,+ C.T.; $\diamond, T_{\alpha}$. Straight lines are the regression lines for G.T. and $T_{\alpha}$, respectively. 
sensitivity of the method. This means that, among these three methods, ultrasound is the only one able to detect a small evolution of the system at the beginning of the aggregation process. The slope of $\Delta \alpha$ in the first step may thus provide information on the kinetics of the aggregation process.

Figure 7 shows the relaxation spectrum i.e. the $\Delta \alpha / f^{2}$ variations as a function of frequency for a $1.45 \%(w / w)$ amylose gel at different ageing times. Here again, this display involved a combination of two gelation experiments since the use of the two pairs of transducers was required. It is to be mentioned that we were not able to obtain these spectra with good accuracy at short times. As an illustration, the curve at $t=12$ minutes coincided with the $X$ axis, $\Delta \alpha$ being very low. The $\Delta \alpha / f^{2}$ traces were well defined at longer times and were dramatically shifted to higher values upon ageing of the amylose gel. The loss of macromolecule mobility during the three steps of the amylose gelation (phase separation, aggregation and crystallization) is clearly responsible for the $\Delta \alpha / f^{2}$ increase. If a single relaxation process was occurring a sharp decrease of $\Delta \alpha / f^{2}$ with frequency would be expected. Before gelation took place $(\mathrm{G} . \mathrm{T} .=26 \mathrm{~min}), \Delta \alpha / f^{2}$ varied very little with frequency. This is consistent with the previous report that no visible relaxation process exists within the frequency range $1-100 \mathrm{MHz}$ in solutions of polysaccharides (11). In the gel state, beyond $60 \mathrm{~min}, \Delta \alpha / f^{2}$ decreased regularly from 20 to $80 \mathrm{MHz}$. Below $20 \mathrm{MHz}, \Delta \alpha / f^{2}$ decreased more significantly. This may correspond to the end of a relaxation process. This result is in good agreement with those reported $(7,10)$ on agarose or carrageenan gels in which a relaxation process at $\sim 10 \mathrm{MHz}$ was found. According to these authors, the strong relaxations observed at these frequencies reflect a property of the overall network structure rather than conformational change of the macro-

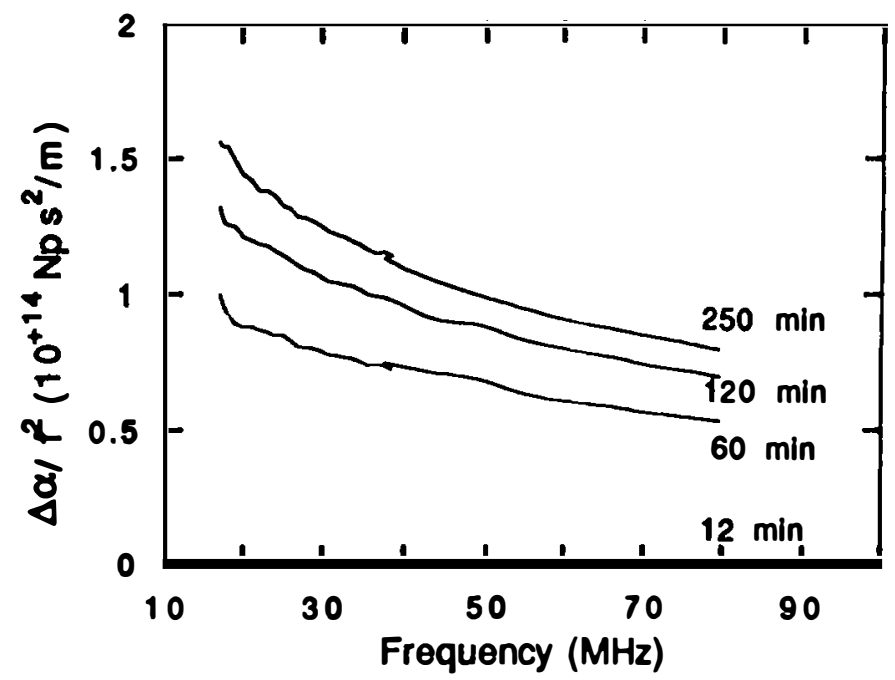

Figure 7 Ultrasonic relaxation spectra for a $1.45 \%$ amylose solution at different times of the gelation process. The results at 12 min almost coincide with the $X$-axis. molecules. They concluded that the most probable mechanism of relaxation is predominantly a result of the motion of solvent molecules within the polymer network. Unfortunately, the frequency range examined here was not large enough for a further comparison. Several relaxation processes having at least one relaxation time or a distribution of relaxation times are perhaps responsible for the decrease of $\Delta \alpha / f^{2}$ between 20 and $80 \mathrm{MHz}$. Several inflection points, for instance at $50 \mathrm{MHz}$ for the 60 and 120 min curves, may correspond to relaxation times characteristic of conformational mobility of macromolecular chains. Aggregation and development of the cross-linked polymer network seem to be more important than structural changes for the relaxation behaviour within the frequency range examined. No more information about the relaxation processes could be obtained from the velocity measurements. No detectable change in the sound velocity during the gelation kinetics could be observed. Whatever the time or frequency considered, the velocity was of $1496 \mathrm{~m} / \mathrm{s}$; this corresponds to the velocity in water at $25^{\circ} \mathrm{C}$ and is easily explained by the fact that the sample contains $99 \%$ of water.

As mentioned above the quantity $\Delta \alpha / f$ is proportional to the loss modulus $M^{\prime \prime}$ which is equal to $K^{\prime \prime}+4 / 3 G^{\prime \prime}$. The frequency dependence of $\Delta \alpha / f$ upon frequency is shown in Figure 8 for a $1.03 \%(\mathrm{w} / \mathrm{w})$ amylose solution in $0.3 \mathrm{~mol} / \mathrm{dm}^{3}$ $\mathrm{KCl} . \Delta \alpha / f$, and hence $M^{\prime \prime}$, shifted along the $Y$-axis when ongoing from the sol to the gel state. This means that the moduli, $K^{\prime \prime}$ and $G^{\prime \prime}$, increased together, but it is not possible to distinguish their respective contributions. From the very beginning of the process to $T_{\alpha}(25 \mathrm{~min}), M^{\prime \prime}$ did not vary with the frequency as illustrated by the horizontal line at $t=25 \mathrm{~min}$. Beyond $T_{\alpha}, \Delta \alpha / f$ showed a linear dependence upon frequency, with a slope increasing progressively. This slope at 120 and $400 \mathrm{~min}$ appeared to reach a limiting value of 0.6. Therefore, the variation of $M^{\prime \prime}$ as a function of

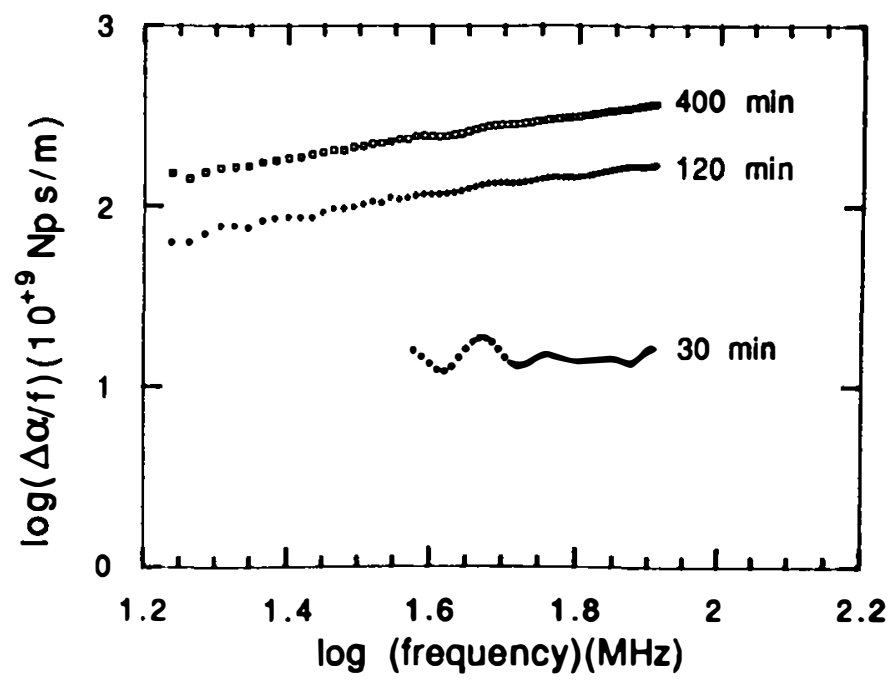

Figure $8 \Delta \alpha / \mathrm{f}$ as a function of frequency at different times of the gelation process (amylose concentration: $1.03 \%$ ). 
frequency can be expressed by a power-law relationship with the exponent $n=0.6$. This value does not vary with time and is close to the values reported in the literature which lie between 0.66 and 0.7 for different systems (24).

\section{Alginate gelation}

In contrast to amylose, alginate gels remain clear during all the gelation process. Therefore, a turbidity analysis is not possible. Figure 9 shows the evolution of the ultrasonic absorption, as measured at $55 \mathrm{MHz}$, during the gelation process of a $0.5 \%$ alginate gel at $25^{\circ} \mathrm{C}$. The EDTA- $\mathrm{Na}_{2}-\mathrm{Ca}$ concentration remained fixed at $0.5 \%$ whereas the GDL concentrations were of $0.35,0.4$ and $0.5 \%$. Overall, the $\Delta \alpha$ trace was comparable to that obtained with amylose. During the induction period (below $T_{\alpha}$ ), which lasted $\sim 80$ min with $0.4 \% \mathrm{GDL}$, for instance, the absorption remained low but increased slightly. The second step was characterized by a sharp increase in $\Delta \alpha$. In the third step, the absorption exhibited a regular rise without reaching a steady state value. First, we checked that this variation of the attenuation cannot be attributed to the evolution of the $\mathrm{pH}$. This is consistent with the results reported in (17) for globular protein solutions. They reported the attenuation as a function of the $\mathrm{pH}$. The only increase in attenuation was observed at the proximity of the isoelectric point, suggesting that variations of the attenuation are the results of structural changes and not of $\mathrm{pH}$ variations. Corresponding $G^{\prime}$ variations as a function of time are shown in Figure 10. Overall, a similar trend as for $\Delta \alpha$ was exhibited and, in contrast to amylose, $T_{\alpha}$ was very close to gel Time (Table 1).

Gelation of alginate is mostly governed by the amount of calcium ions that can be chelated by the macromolecular chains through guluronic sequences. At the beginning of the experiment, at $\mathrm{pH} \sim 6$, all the calcium ions are

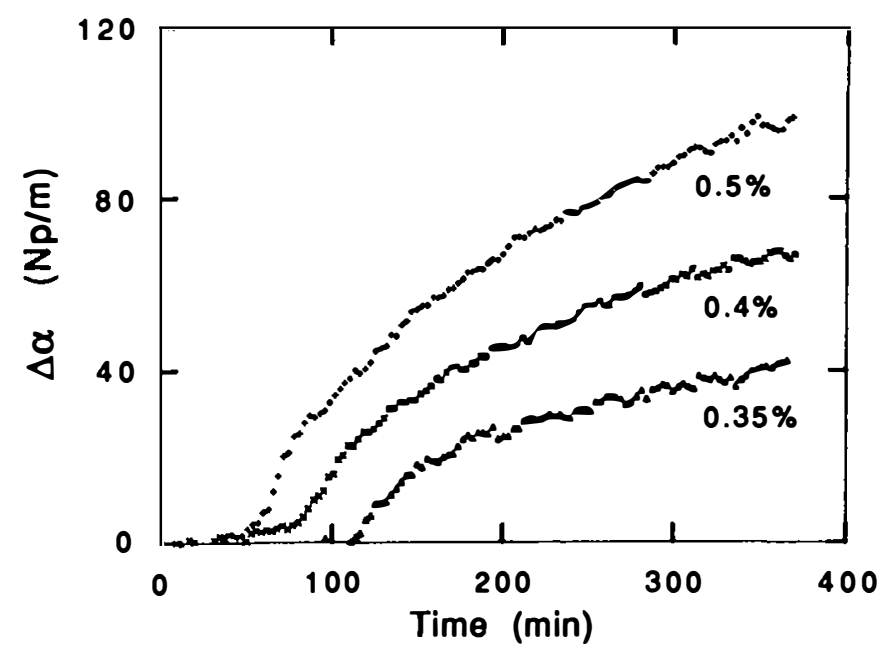

Figure $9 \Delta \alpha$ variations during gel formation of alginate for three GDL concentrations: $\Delta, 0.35 ; \times, 0.4 ;+, 0.5 \%$ (Alginate concentration: $0.5 \%$; EDTA concentration: $0.5 \%$ ). complexed by EDTA. Their freeing can be progressively achieved as the $\mathrm{pH}$ is lowered. The overall pattern of Figures 9 and 10 was indeed the result of the $\mathrm{pH}$ decrease yielded by the spontaneous hydrolysis of GDL to gluconic acid. As soon as GDL was added, the $\mathrm{pH}$ decreased from its initial value of 6 , first rapidly and then more slowly to reach a constant value after $350 \mathrm{~min}$. The $\mathrm{pH}$ value at this time was dependent on the initial concentration of GDL. A $\mathrm{pH}$ of $4.49,4.43$ and 4.32 was obtained for GDL concentrations of $0.35,0.40$ and $0.50 \%$ respectively. Clearly, the GDL concentration defines the rate of the $\mathrm{pH}$ decrease as well as the final $\mathrm{pH}$. It infers that the rate of the $\mathrm{Ca}^{2+}$ release from EDTA-Na-2-Ca and the amount of $\mathrm{Ca}^{2+}$ available for chelation with alginate is effected by the GDL concentration. We observed that, for the three experiments, the second step of the process, as characterized by $T_{\alpha}$ and G.T., started when the $\mathrm{pH}$ was equal to 5 . We could estimate the amount of calcium ions freed from EDTA on the basis of the equilibrium constant of EDTA-Na $-\mathrm{Ca}$ and of the acidity constants of the acidic form of EDTA, a tetraacid, as described in (25). If we define by $x$ the degree

Table 1 Comparison of $T_{\alpha}$ and gel time for alginate

\begin{tabular}{lcc}
\hline $\begin{array}{l}\text { GDL concentration } \\
(\%, w / w)\end{array}$ & $\begin{array}{l}T_{\alpha} \\
(\mathrm{min})\end{array}$ & $\begin{array}{l}\text { Gel time } \\
(\mathrm{min})\end{array}$ \\
\hline & & \\
0.5 & 63 & 69 \\
0.45 & 70 & 79 \\
0.4 & 80 & $\mathrm{n} . \mathrm{d}$. \\
0.35 & 111 & 115 \\
0.30 & 135 & 143 \\
\hline
\end{tabular}

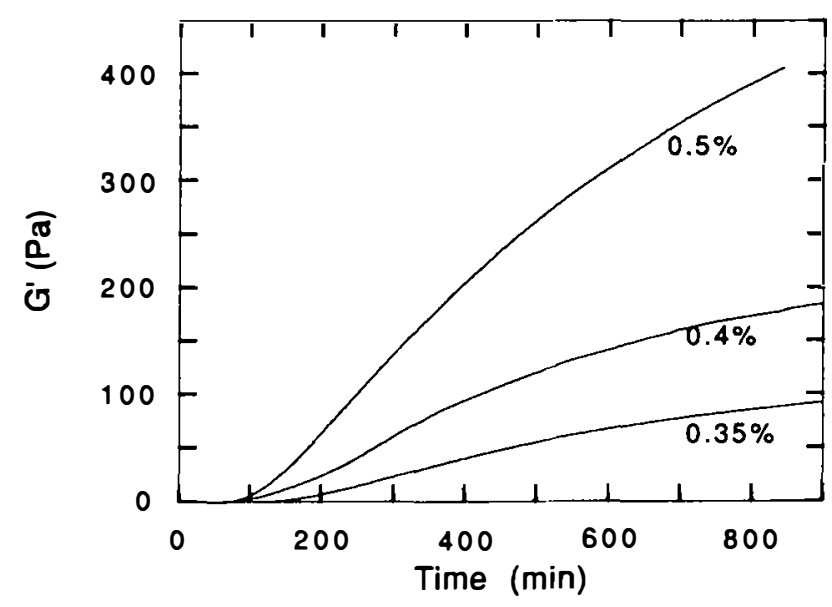

Figure $10 \mathrm{G}^{\prime}$ variations as a function of time during gelation for the three gelling systems studied in Figure 9. 
of complexation of calcium with EDTA, the following relationship applies:

$$
\frac{(1-x)^{2}}{x}=\frac{K}{C} \frac{\left(\mathrm{H}_{3} \mathrm{O}^{+}\right)^{2}}{K_{4} K_{3}}
$$

with:

\section{$C:$ EDTA- $\mathrm{Na}_{2}-\mathrm{Ca}$ molar concentration}

$K_{4}$ and $K_{3}$ two of the acidity constants of the acidic form of EDTA $\left(\mathrm{pK}_{4}=6.2\right.$ and $\left.\mathrm{pK}_{3}=10.3\right)$,

$K$, the equilibrium constant of EDTA- $\mathrm{Na}_{2}-\mathrm{Ca}$

$$
\left(K=1.09610^{-11}\right) \text {. }
$$

As a rule, the amount of freed calcium ions for this EDT A content $\left(1.3410^{-2}\right.$ mole $)$ would be of $0.5 \%$ at $\mathrm{pH}=$ 6 , of $5 \%$ at $\mathrm{pH}=5$ and of $60 \%$ at $\mathrm{pH}=4$. Clearly, as soon as the $\mathrm{pH}$ is $<5$, the amount of freed calcium cations is great enough $(\sim 5 \%)$ to create interchains association between polyguluronic strands. Therefore, the increase of $\Delta \alpha$ beyond $T_{\alpha}$, and of $G^{\prime}$ beyond G.T., is the result of additional crosslinking, or dimerization, of alginate molecules. After $350 \mathrm{~min}$, the absorption was almost three times greater for an alginate gel containing $0.5 \%$ of GDL than for one with $0.35 \%$ of GDL. From the corresponding $\mathrm{pH}$ values at $350 \mathrm{~min}$ (see above) and using the abovementioned procedure, we could estimate the respective amounts of calcium ions freed at this time: $17 \%$ with $0.35 \%$ GDL, $19 \%$ with $0.4 \%$ GDL and $25 \%$ with $0.5 \%$ GDL. It was during the first step that the $\mathrm{pH}$ varied most, from $\sim 6$ to $\sim 5$, but the amount of $\mathrm{Ca}^{2+}$ freed was relatively low. On another hand, the $\mathrm{pH}$ values at the end of the experiment, despite being quite close, yielded largely different amounts of freed calcium. This may explain the GDL concentration dependence of final values of $G^{\prime}$ and $\Delta \alpha$. It is therefore clear that for alginate gelation, ultrasonic measurements provide the same type of information as rheological measurements in oscillatory shear.

\section{Conclusion}

The present work has shown that ultrasonic spectroscopy can be a useful and powerful tool to monitor the gelation of polysaccharides. The attenuation is sensitive to small structural changes in polysaccharide solution even if there is $\sim 99 \%$ of water. For both systems, the onset of gelation is clearly detected. With amylose, the ultrasonic lag time $T_{\alpha}$ is shorter than the G.T. This means that ultrasound has been able to detect the phase separation in its first step, well before the evolution of the viscoelastic properties. It is clear that this technique is more sensitive than rheological methods in this respect. It appears even more sensitive than the turbidity measurements. For alginate, $T_{\alpha}$ and G.T. are close to each other, probably because there is no phase separation. Unfortunately, whatever the type of gelling system, no variation in the propagation velocity could be recorded. Therefore, it was impossible to evaluate the storage modulus $M^{\prime}$ which would be more pertinent to evaluate the evolution in the elastic properties of such systems. More work at lower frequencies $(<10 \mathrm{MHz})$ is needed in order to detect relaxation processes but at these frequencies the attenuation is weak and it is difficult to record variations in the signal with good accuracy. Nevertheless, accurate velocity and attenuation measurements should be made over a wide range of frequency. In addition, it would be interesting to perform studies on other gelling systems and to develop shear waves measurements. It would be easier to relate $G^{\prime}$ and $G^{\prime \prime}$ obtained from these measurements to rheological methods.

\section{References}

1. Javanaud,C. (1988) Ultrasonics, 26, 117-123.

2. Povey,M.J.W. and McClements,D.J. (1988) J. Food Engng, 8, 217-245.

3. McClements,D.J., Proc. Ultrasonics International 91, Butterworth-Heinemann, Oxford, UK, pp. 103-106.

4. McClements,D.J., Povey,M.J.W. and Dickinson,E., Proc. Ultrasonics International 91, ButterworthHeinemann, Oxford, UK, pp. 107-110.

5. Howe,A.M. and Robins,M.M. (1990) Colloids Surf., 43, 83-94.

6. Lee,H.O., Luan,H. and Daut,D.G. (1992) J. Food Engng, 16, 127-150.

7. Gormally,J., Pereira,M.C. and Wyn-Jones,E. (1982) J. Chem. Soc., Faraday Trans. 2, 78, 1661-1673.

8. Gettins,W.J., Jobling,P.L. and Wyn-Jones,E. (1978) J. Chem. Soc., Faraday Trans. 2, 74, 1246-1252.

9. Pass,G., Phillips,G.O., Wedlock,D.J. and Wyn-Jones, E. (1978) Macromolecules, 11, 433-435.

10. Pereira,M.C. and Wyn-Jones,E. (1982) Carbohydr. Polym., 2, 103-113.

11. Kato,S., Suzuki,T., Nomura,H. and Miyahara,Y. (1980) Macromolecules, 13, 889-892.

12. Miles,M.J., Morris,V.J. and Ring,S.G. (1985) Carbohydr. Res., 135, 257-269.

13. Ellis,H.S. and Ring,S.G. (1985) Carbohydr. Polym., 5, 201-213.

14. Morris,E.D., Rees,D.A. and Thom,D. (1978) Carbohydr. Res., 66, 145-154.

15. Thom,D., Grant,G.T., Morris,E.D. and Rees,D.A. (1982) Carbohydr. Res., 100, 29-42.

16. Lairez,D., Durand,D. and Emery,J.R. (1991) Makromol. Chem., Macromol. Symp., 45, 31-45.

17. Pavlovskaya,G., McClements,D.J. and Povey,M.J.W. (1992) Food Hydrocoll., 6, 253-262.

18. Doublier,J.L. and Choplin,L. (1989) Carbohydr. Res., 193, 215-226.

19. Emery,J.R. (1986) Annales des composites, 89-108.

20. Emery,J.R. and Tabellout,M. (1990) Revue Phys. Appl., 25, 243-251.

21. Holmes,A.K., Challis, R.E. and Wedlock,D.J. (1993) J. Coll. Interf. Sci., 156, 261-268. 
22. Doublier,J.L., Llamas,G. and Choplin,L. (1990) Makromol. Chem., Macromol. Symp., 39, 171-177.

23. Clark,A.H., Gidley,M.J., Richardson,R.K. and RossMurphy,S.B. (1989) Macromolecules, 22, 346-351.

24. Lairez,D. (1990) Université du Maine, Le Mans, France.

25. Bouffard-Roupe,C. (1989) Thesis. Université J.

Fourrier, Grenoble I, France. 